LETTER TO EDITOR

\title{
Clinical and Demographic Characteristics of COVID-19 Deceased Patients in An Iranian Referral Center
}

\author{
Parnian Shobeiri ${ }^{1}$, Mehrdad Hasibi ${ }^{2}$, Hamed Abdollahi ${ }^{3}$, Mitra Hajinoori $^{2}$, Maryam Taghizadeh ${ }^{4}$, \\ Maryam Moosivand ${ }^{5}$, Mina Abdolahi ${ }^{6}$, Ali Asadollahi-Amin ${ }^{7}$ \\ ${ }^{1}$ School of Medicine, Tehran University of Medical Sciences, Tehran, Iran \\ ${ }^{2}$ Department of Infectious Diseases, Amir-Alam Hospital, Tehran University of Medical Sciences, Tehran, Iran \\ ${ }^{3}$ Department of Anesthesiology, Amir Alam Hospital Complexes, Tehran University of Medical Sciences, Tehran, Iran \\ ${ }^{4}$ Department of Surgery, Amir Alam Hospital Complexes, Tehran University of Medical Sciences, Tehran, Iran \\ ${ }^{5}$ Department of Emergency, Amir Alam Hospital Complexes, Tehran University of Medical Sciences, Tehran, Iran \\ ${ }^{6}$ Department of Nutrition, Amir Alam Hospital Complexes, Tehran University of Medical Sciences, Tehran, Iran \\ ${ }^{7}$ Iranian Research Center for HIV/AIDS, Iranian Institute for Reduction of High-Risk Behaviors, Tehran University of \\ Medical Sciences, Tehran, Iran
}

\begin{abstract}
After more than one year, the Novel Corona Virus (COVID-19) pandemic is still going on. In this retrospective study, we reported clinical characteristics of patients who died cause of COVID-19. A total of 32 (7.6\%) deaths were recorded among 422 patients with COVID-19, of which 280 (66.3\%) patients were male and $142(33.7 \%)$ female. Males had a significantly higher death incidence than females $(26 / 32,81.25 \%$ vs. $6 / 32,18.75 \%$, respectively; P-value $>0.001)$. Hypertension $(14 ; 43.8 \%)$ and Diabetes Mellitus (13; 40.6\%) were the most common independent comorbidities among patients. Other risk factors are heart disease, renal disease, chemotherapy, and corticosteroid therapy. Dyspnea and cough were the most frequent symptom of admission. Increased levels of ESR and creatinine are significantly associated with mortality. J Microbiol Infect Dis 2021; 11(4):238-240.
\end{abstract}

Keywords: COVID-19, Clinical characteristics, Demographic characteristics, Mortality, Iran

Dear Editor,

Novel Corona Virus (COVID-19) firstly appeared as an epidemic condition in Wuhan, China, on December 31, 2019. Then, as of January 30 , the World Health Organization Emergency Committee declared that the Coronavirus outbreak developed a Public Health Emergency of International Concern, respectively, a pandemic. As of August 21, 2021, there are more than 212 million confirmed cases of COVID-19 worldwide, with approximately $4,435,620$ deaths $[1,2]$.

The novelty of COVID-19 and its incredibly diverse clinical features, manifestations from asymptomatic to severe respiratory insufficiency conditions, made this circumstance hard to manage, as we do not know the exact mechanism of pathogenicity of this new disease. Hence, in this situation, researchers and studies help to handle the problem better than before. Nevertheless, studies showed divergence estimating the risk factors of death due to COVID-19. Moreover, researchers should conduct more studies to reveal the unknown characteristics of this current disease [3].

On February 20, 2020, the Islamic Republic of Iran reported the first two cases who died due to COVID-19 in Qom Province. Thus, Iran was the first middle-eastern country, which infected by COVID-19. Presently, Iran announced $4,677,114$ confirmed cases, and about 102,038 died [2]. As the lack of enough data describing unrevealed aspects of the pandemic in Iran, we performed a retrospective cohort study to investigate the clinical features of 32 patients deceased of

Correspondence: Ali Asadollahi-Amin, MD, Iranian Research Center for HIV/AIDS, Imam Khomeini Hospital Complex, 
COVID-19 admitted to a referral COVID-19 hospital in Tehran.

We included in this pilot study 422 confirmed COVID-19 patients with Reverse Transcription Polymerase Chain Reaction (RT-PCR) test, referred to Amir Alam Hospital, Tehran, Iran, from February 20 to March 20, 2020. The Ethics Commission of the Hospital waived written informed consent for emerging infectious diseases. Therefore, we used particular codes for each patient to anonymize the identification, and all their related data remained intimate during the study.

The Ethics Committee of Tehran University of Medical Sciences, Tehran, Iran approved this study (IR.TUMS.VCR.REC.1399.088, available at ethics.research.ac.ir).

Our team in Amir Alam hospital hospitalized COVID-19 patients based on the COVID-19 national guideline; high-risk patients (immunocompromised patients and patients with comorbidities including hypertension, diabetes, ischemic heart diseases, chronic lung diseases, chronic kidney diseases, and chronic liver disease) representing moderate symptoms, and severe cases (pneumonia, hypoxemia/ oxygen saturation ( $\mathrm{sO} 2, \%)<93 \% /$ $\mathrm{RR}>30$ per minute, respiratory failure, sepsis, and septic shock). In addition, the basic clinical data, including demographical data, laboratory, and radiology results from the electronic medical registry system, were retrieved. In addition, the other clinical data from the hospital documents, including clinical signs, symptoms, and comorbidities, were obtained.

On the first day of admission, the vital signs and clinical symptoms were examined. The axillary temperature of more than $37.3^{\circ} \mathrm{C}$ was considered as fever. Moreover, laboratory results were gathered upon the hospitalization and the last day of life. All patients had at least one spiral chest Computed Tomography (CT) scan without contrast on the first day of admission. A pulmonologist and a radiologist separately interpreted them all. The severity of pulmonary implication based on chest CT scan results was measured. The involvement of both lungs $\leq 25 \%, 26-50 \%$, and $>50 \%$ were referred to as mild, moderate, and severe, respectively.

A total of $32(7.6 \%)$ deaths were recorded among 422 patients with COVID-19; 280
(66.3\%) were male patients, and 142 (33.7\%) were female.

The mean $\pm S D$ age of the patients was $66.62 \pm$ 20.68 years old. Males had a significantly higher death incidence than females (26/32, $81.25 \%$ vs. $6 / 32,18.75 \%$, respectively; p value $>0.001)$.

Comorbidities were present in nearly $75 \%$ of patients. Hypertension (14; 43.8\%) and diabetes $(13 ; 40.6 \%)$ were the most common comorbidities among the patients. Other risk factors are heart disease $(7 ; 21.9 \%)$, renal disease $(2 ; 6.3 \%)$, chemotherapy $(1 ; 3.1 \%)$ and corticosteroid therapy $(1 ; 3.1 \%)$ (Figure 1). Seven patients had two or more risk factors.

The most common symptoms on the first visit were dyspnea $(26 ; 81.3 \%)$, cough $(17 ; 53.1 \%)$, fever $(14 ; 43.8 \%)$ and weakness $(10 ; 31.3 \%)$ (Figure 1).

Only $6.25 \%$ of patients had blood $\mathrm{O} 2$ saturation $(\mathrm{SaO} 2) \geq 90 \%$ without respiratory support on admission. The mean \pm SD of PSO2 without respiratory aid devices was $81.53 \pm 9.88$ on admission, while on the last day of life, this value was found an average of 74.15 with respiratory aid devices.

Venous blood gas (VBG) evaluation showed that the mean of the partial pressure of carbon dioxide $(\mathrm{PCO} 2)$ and $\mathrm{pH}$ were $42.20 \pm 9.33$ and $7.30 \pm 08$, respectively. Before intubation, $31.3 \%$ of the patients did not have any respiratory support, $3.1 \%$ had nasal cannula, $15.6 \%$ received O2 with a simple mask, $46.9 \%$ with reserve mask, and $3.1 \%$ used continuous positive air pressure machines (CPAP).

Approximately $84.37 \%$ of patients (27/32) were intubated, of which $40.62 \%$ were within 48 hours after hospitalization. The most common imaging sign observed on their chest CT scan was ground-glass opacities $(68.8 \%)$ and consolidation (25\%). In addition, in $6.3 \%$ of patients, bilateral severe pulmonary infiltration was reported, while moderate and mild lung involvement were both $3.1 \%$ (Table 1). The WBC and lymphocyte were in the normal range, while creatinine, ESR, and CRP were higher than the normal range (Table 2).

Our study identifies various risk factors causing death in patients hospitalized in a referral hospital, Tehran, Iran. Among them, hypertension and diabetes were commonly the 
most prevalent comorbidities in patients who died due to the COVID-19 infection.

Table 1. The Breathing support, Intubation time, and Radiological manifestation in the participants.

\begin{tabular}{lcc}
\hline Findings & Results & N (\%) \\
\hline Breathing support & none & $10(31.3)$ \\
& nasal & $1(3.1)$ \\
& mask & $5(15.6)$ \\
& Reserved mask & $15(46.9)$ \\
& CPAP & $1(3.1)$ \\
Intubation time (d) & $3.27 \pm 2.28$ \\
Radiological & Ground class & $22(68.8)$ \\
manifestation & consolidation & $8(25)$ \\
& Nodule & $5(12.5)$ \\
\hline
\end{tabular}

Table 2. Laboratory tests in the participants.

\begin{tabular}{lc}
\hline Laboratory Tests & Values \\
\hline WBC $\left[{ }^{*} 10^{\wedge} 3 / \mu \mathrm{L}\right]$ & $7.93 \pm 4.76$ \\
Lymphocyte $\left[{ }^{*} 10^{\wedge} 3 / \mu \mathrm{L}\right]$ & $14.86 \pm 8.83$ \\
Creatinine $[\mathrm{mg} / \mathrm{dL}]$ & $1.45 \pm 0.59$ \\
ESR $\left[\mathrm{mm} / 1^{\mathrm{st}}\right.$ Hour] & $56.56 \pm 20.28$ \\
CRP $[\mathrm{mg} / \mathrm{dL}]$ & $90.54 \pm 30.68$ \\
\hline
\end{tabular}

Predominantly, the male gender has higher rates of death. Additionally, dyspnea and cough were the most frequent symptom of admission. We found a significant association between mortality and high ESR and creatinine levels $(p=0.01)$. We did not observe a significant increasing trend of $d$-dimer on admission and the last day of life. However, consistent with a previous study, the deceased cases had a high level of d-dimer, which was above $1 \mu \mathrm{g} / \mathrm{ml}$ on admission and the last day of life [4].

In this study, severe respiratory disorders manifestations such as dyspnea, $\mathrm{SO}_{2}<90 \%$, and respiratory acidosis were permanent up to death. A review article shows that metabolic acidosis occurs in severe COVID-19 patients due to invasive ventilating [5]. Additionally, studies report cases with respiratory acidosis that have been corrected [6].

Due to the retrospective nature of our study, we could not obtain detailed clinical and microbiological data of patients. Therefore, further prospective cohort studies are needed to reveal the other risk factors associated with mortality compared to those who survived.

Moreover, other studies imply that fever, fatigue, and dry cough are the most common clinical manifestations in COVID-19 patients [7]. Zhang $\mathrm{JJ}$ et al. show that lymphopenia is common in patients [8]. Noticeably, based on the binomial test, intubation and ICU admission time distribution were significantly associated with death incidence $(P=0.03$ and $\mathrm{P}<0.05$, respectively).

In conclusion, similar to previous studies, hypertension and diabetes were the most common comorbidities among deceased COVID-19 patients. However, further prospective cohort studies are warranted.

\section{ACKNOWLEDGMENTS}

Declaration of conflicting interest: The author(s) declare no potential conflicts of interest concerning this article's research, authorship, and/or publication.

Financial disclosure: No financial support was received for this study

\section{REFERENCES}

1. Phelan AL, Katz R, Gostin LO. The novel coronavirus originating in Wuhan, China: challenges for global health governance. JAMA 2020; 323:709-710.

2. https://www.worldometers.info/coronavirus. [updated 21 August 2021; cited 21 August 2021]. https://www.worldometers.info/ coronavirus/country/iran/

3. Huang C, Wang Y, Li X, et al. Clinical features of patients infected with 2019 novel coronavirus in Wuhan, China. Lancet. 2020; 395:497-506.

4. Zhou F, Yu T, Du R, et al. Clinical course and risk factors for mortality of adult inpatients with COVID-19 in Wuhan, China: a retrospective cohort study. Lancet. 2020; 395:1054-1062.

5. Xie P, Ma W, Tang H, Liu D. Severe COVID-19: a review of recent progress with a look toward the future. Front Public Health. 2020; 8:189.

6. Levin MA, Shah A, Shah R, et al. Differential ventilation using flow control valves as a potential bridge to full ventilatory support during the COVID-19 crisis: from bench to bedside. Anesthesiology 2020; 133:892-904.

7. Wang $\mathrm{D}, \mathrm{Hu} \mathrm{B}, \mathrm{Hu} \mathrm{C}$,et al. Clinical characteristics of 138 hospitalized patients with 2019 novel coronavirus-infected pneumonia in Wuhan, China. JAMA 2020; 323:1061-1069.

8. Zhang JJ, Dong X, Cao YY,et al. Clinical characteristics of 140 patients infected with SARS-CoV-2 in Wuhan, China. Allergy 2020; 75:1730-1741. 